\title{
Liste des membres
}

Volume 34, 1967

URI : https://id.erudit.org/iderudit/1007426ar

DOI : https://doi.org/10.7202/1007426ar

Aller au sommaire du numéro

Éditeur(s)

Les Éditions Historia Ecclesiæ Catholicæ Canadensis Inc.

ISSN

0318-6172 (imprimé)

1927-7067 (numérique)

Découvrir la revue

Citer ce document

(1967). Liste des membres. Sessions d'étude - Société canadienne d'histoire de l'Église catholique, 34, 87-96. https://doi.org/10.7202/1007426ar

Tous droits réservés @ Les Éditions Historia Ecclesiæ Catholicæ Canadensis Inc., 1968
Ce document est protégé par la loi sur le droit d'auteur. L'utilisation des services d'Érudit (y compris la reproduction) est assujettie à sa politique d'utilisation que vous pouvez consulter en ligne.

https://apropos.erudit.org/fr/usagers/politique-dutilisation/ 


\section{MEMBRES BIENFAITEURS *}

1938 - M. le chanoine Eugène Carrier, curé de Notre-Dame, Lévis, P.Q.

1942 - Son Excellence $M^{\mathrm{gr}}$ Arthur Douville, Evêque de Saint-Hyacinthe, Evêché de Saint-Hyacinthe, Saint-Hyacinthe, P.Q.

1944 - M. le chanoine Robert Charland, Séminaire de Nicolet, Nicolet, P.Q.

1945 - Le très révérend Père Provincial des Missionnaires Oblats de Marie-Immaculée, 3456, avenue Ontario, Montréal 25, P.Q.

1945 - L'honorable Alphonse Raymond, 1321, rue Sherbrooke ouest, Montréal, P.Q.

1946 - M. l'abbé Adrien Verrette, 517, rue Pine, Manchester, N.H., E.-U.

1949 - M. J.-E. Guertin.

1950 - Son Excellence $\mathbf{M}^{\mathrm{gr}}$ Joseph-Arthur Papineau, Evêché de Joliette, Joliette, P.Q.

1958 - Son Excellence $\mathrm{M}^{\mathrm{gr}}$ Gérard-Marie Coderre, Evêque de Saint-Jean, Saint-Jean, P.Q.

\section{MEMBRES DONATEURS *}

1945 - M. Conrad Charlebois, C.O.St-G.G., 70, rue Maurice, Hull, P.Q.

1945 - M. C.-J. Hubert, 1490, avenue Laframboise, Saint-Hyacinthe, P.Q.

1945 - M. le major Gustave Lanctot.

1945 - M. le notaire Victor Morin.

1945 - M. Joseph-Edouard Perrault.

1945 - La révérende Mère Provinciale des Sœurs de la Présentation, Saint-Hyacinthe, P.Q.

1945 - $\mathbf{M}^{\mathbf{g r}}$ Albert Tessier, Séminaire des Trois-Rivières, Trois-Rivières, P.Q.

1945 - Le très révérend Père Recteur de l'Université d'Ottawa, Ottawa, Ont.

1949 - Son Excellence $\mathbf{M}^{\mathrm{gr}}$ Georges Cabana, Archevêque de Sherbrooke, Sherbrooke, P.Q.

1949 - M. C.-R. Dufault, 505, rue Langevin, Saint-Boniface, Man.

1953 - M. Gustave Bellefleur, 3973, rue Mentana, Montréal, P.Q.

1958 - Son Excellence $\mathrm{M}^{\mathrm{gr}}$ Charles-Eugène Parent, Archevêché de Rimouski, Rimouski, P.Q.

* On peut devenir bienfaiteur en versant la somme de cent dollars, membre donateur en versant cinquante dollars. 
1958 - Sœurs de la Charité, La supérieure générale des, Hôtel-Dieu, Saint-Hyacinthe, P.Q.

1958 - M. l'abbé Emile Turmel, Ancienne Lorette, P.Q.

1963 - M. l'abbé Charles Michaud, Maison mère des Søurs du SaintRosaire, Rimouski, P.Q.

1964 - Cournoyer, M. l'abbé Georges-Henri, Presbytère Saint-Joseph, Saint-Hyacinthe, P.Q.

1964 - Tremblay, $M^{\mathrm{gr}}$ Victor, P.D., Petit Séminaire, Chicoutimi, P.Q.

\section{MEMBRES}

Abbaye de Notre-Dame de Nazareth, L', Rougemont, P.Q.

Académie de Québec, La Bibliothèque de l', 2410, chemin Sainte-Foy, Québec 10, P.Q.

Agnès-de-Jésus, n.d.b.c., Rév. Sœur, Ecole Sainte-Claire, ChicoutimiNord, P.Q.

American Bibliographical Center, 800 East Micheltorena St., Santa Barbara, California, U.S.A.

Anne-Marie, n.d.b.c., Rév. Søur, Maison-Mère du Bon Conseil, Chicoutimi, P.Q.

Archambault, M. le docteur A.-D., 4600, boulevard LaSalle, Verdun, P.Q. Archives acadiennes, Bibliothèque générale, Université de Moncton, Moncton, N.B.

Archives du Monastère, Les Hospitalières de Saint-Augustin, 75, rue des Remparts, Québec 4, P.Q.

Archives Publiques du Canada, La Bibliothèque des, rue Sussex, Ottawa, Ont.

Archiviste du Québec, L', Parc des Champs de Bataille, Québec, P.Q.

Baillargeon, M. l'abbé Noël, Séminaire de Québec, C.P. 460, Québec, P.Q.

Barabé, Le R.P. P.H., o.m.i., 3390, chemin Saint-Louis, Québec 10, P.Q.

Baudry, Le R.P. René, c.s.c., Ambassade du Canada, 35, avenue Montaigne, Paris VIII', France.

Beaudin, M. l'abbé François, 2000 ouest, rue Sherbrooke, Montréal 25, P.Q.

Beaudry, Rev. A.-T., 50 Melville, Pittsfield, Mass., U.S.A.

Beaulieu, M. Gaston, suite 816, 243, boulevard Cassandra, Don Mills, Ont.

Beaulieu, M. le chanoine Henri, Séminaire de Québec, C.P. 460, Québec, P.Q.

Beaulieu, M. Wilfrid, Le Travailleur, case postale 195, Worchester 1, Mass., U.S.A.

Behrer, M. W., C.R., Président, Commission des Ecoles catholiques, 1460, chemin Sainte-Foy, Québec 6, P.Q.

Bélanger, Mlle Françoise, 5373, rue Durocher, Outremont, P.Q.

Bélanger, $\mathrm{M}^{\mathrm{gr}}$ Léon, p.d., curé, L'Islet, P.Q.

Bélanger, $\mathrm{M}^{\mathrm{gr}}$ René, P.D., 980, rue Richelieu, Québec, P.Q.

Bélanger, Son Excellence $M^{\text {gr }}$ Valérien, 1071, rue Cathédrale, Montréal 3, P.Q. 
Bertrand, l'honorable M. Lionel, C.P. 190, Sainte-Thérèse-de-Blainville, P.Q.

Bérubé, M. le chanoine Léo, Archevêché de Rimouski, Rimouski, P.Q.

Bibeau, R.P. Hector, s.m.m., Séminaire Montfort, Papineauville, P.Q.

Biblioteca Apostolica Vaticana, Cité du Vatican.

Bibliothécaire, Le R.P., Couvent des Pères Dominicains, 96, avenue Empress, Ottawa 4, Ont.

Bibliothécaire, Le R.P., Abbayé Saint-Benoît-du-Lac, Qué.

Bibliothécaire, Rév. Sr, 1190, rue Guy, Montréal 35, P.Q.

Bibliothèque, Collège Basile-Moreau, 845, rue Sainte-Croix, Montréal 9, P.Q.

Bibliothèque, Collège de Saint-Boniface, 200, avenue de la Cathédrale, Saint-Boniface, Man.

Bibliothèque, Université Laurentienne, Sudbury, Ont.

Bibliothèque de l'Assemblée législative de la Province de Québec, La, Hôtel du Gouvernement, Québec, P.Q.

Bibliothèque de la ville de Montréal, La, 1210 est, rue Sherbrooke, Montréal 24, P.Q.

Bibliothèque du Parlement, La, Hôtel du Gouvernement, Ottawa, Ont.

Bibliothèque Saint-Sulpice, La, 1700, rue Saint-Denis, Montréal 18, P.Q.

Bibliothèque des Trois-Rivières, Case postale 1207, 1220, rue JeanNicolet, Trois-Rivières, P.Q.

Bibliothèque du Séminaire, C.P. 410, Joliette, P.Q.

Bibliothèque du Séminaire de Sherbrooke, C.P. 790, rue Marquette, Sherbrooke, P.Q.

Billy, M. J.-Louis, C.0.St-G.G., 425, rue Saint-Patrice, Ottawa 1, Ont.

Bourassa, M. le curé Lionel, Lavigne (Nipissing), Ont.

Brouillard, $\mathrm{M}^{\mathrm{gr}}$ Armand, P.D., Séminaire, C.P. 370, St-Hyacinthe, P.Q.

Bruchési, Son Excellence M. Jean, Ambassade du Canada, Casilla de Coreo 1598, Buenos Aires, Argentine.

Bujanda, M. l'abbé Jésus-M., Faculté des Arts, Université, Sherbrooke, P.Q.

Bureau des Archives, Sœurs de l'Assomption de la Sainte-Vierge, Nicolet, P.Q.

Campeau, M. le curé 0., Coniston, Ont.

Capucins, Les révérends Pères, 460, $8^{\circ}$ avenue, Québec 3, P.Q.

Carleton University, The Library, Colonel By Drive, Ottawa, Ont.

Carrière, Le R.P. Gaston, o.m.i., Séminaire universitaire, 249, rue Main, Ottawa, Ont.

Cartier, M. le curé Lucien, Saint-Aimé (Richelieu), P.Q.

Chabot, R.F. Alcide, f.m.s., Scolasticat central de Montréal, 7000, rue Marie-Victorin, Montréal 39, P.Q.

Charbonneau, M. Louis, 1, rue Beckwith, Ottawa, Ont.

Charbonneau, M. Rhéal, Secrétaire national de l'Action catholique canadienne, 3827, rue Saint-Hubert, Montréal, P.Q.

Charron, M. le curé Léo, Pain Court, Ont.

Chartrand, M. le curé Roland, 369, Grande Côte, Sainte-Thérèse-Ouest, Terrebonne, P.Q.

Chaussé, R.P. Gilles, s.j., 1800 est, boulevard Henri-Bourassa, Montréal 12, P.Q. 
Clercs de Saint-Viateur, La Maison Provinciale des, a/s Le Bibliothécaire, 1145, rue Saint-Viateur, Outremont, P.Q.

Collège Bourget, La Bibliothèque, Rigaud, P.Q.

Collège des Jésuites, Le, 1150 ouest, rue Saint-Cyrille, Québec, P.Q.

Collège de Jésus-Marie de Sillery, Le, 2047, chemin Saint-Louis, Québec 6, P.Q.

Collège de Lévis, $\mathbf{M}^{\mathrm{gr}}$ le Supérieur du, Lévis, P.Q.

Collège Saint-Laurent, a/s R.P. Bibliothécaire, 625, boulevard SainteCroix, Ville Saint-Laurent, Montréal 9, P.O.

Collège Sainte-Anne-de-la-Pocatière, La Bibliothèque du, Sainte-Anne-dela-Pocatière, P.Q.

Collège Saint-Maurice, La Directrice du, 640, rue Girouard. SaintHyacinthe, P.Q.

Compagnie de Jésus, Le très révérend Père Provincial de la, 3215, chemin Sainte-Catherine, Montréal 26, P.Q.

Comptois, M. le docteur B., 36, rue Elm est, Sudbury, Ont.

Conférence Catholique Canadienne, M. le Secrétaire de la, 90, rue Parent, Ottawa, Ont.

Congrégation de Notre-Dame, Le Bureau des Archives de la, 3040 ouest, rue Sherbrooke, Montréal 25, P.Q.

Cormier, Le R.P. Clément, c.s.c., Université de Moncton, Moncton, N.-B.

Corriveau, M. l'abbé Robert, Napierville (Comté Napierville), P.Q.

Cossette, Le R.P. Joseph, s.j., Collège Saint-Ignace, 1700 est, boulevard Henri-Bourassa, Rosemont, Montréal 12, P.Q.

Dansereau, M. Antoine, p.s.s., Collège de Montréal, 1931 ouest, rue Sherbrooke, Montréal, P.Q.

DeBlois, M. le curé Alexandre, V.F., Sainte-Croix (Lotbinière), P.Q.

Décosse, Son Excellence $\mathbf{M}^{\mathrm{gr}}$ Aimé, Evêque de Gravelbourg, Gravelbourg, Sask.

DeMoissac, La révérende Sœur E., s.g.m., 5l, rue Despins, SaintBoniface 5, Man.

Deschâtelets, Le T.R.P. Léo, o.m.i., Supérieur général, 290, via Aurelia, Rome, Italie.

Desjardins, Le révérend Père Paul, s.j., Collège Sainte-Marie, 1180, rue Bleury, Montréal 2, P.Q.

Desjardins, Le R.P. Léopold, c.ss.r., Mont Saint-Joseph, 161, rue Principale, Aylmer-Est, P.Q.

Dionne, M. J.-A., Ingénieur professionnel, 760, avenue Wiseman, Outremont, P.Q.

Dominicains, Le Bibliothécaire du monastère des révérends Pères, 175 ouest, Grande Allée, Québec 6, P.Q.

Dominicains, Le couvent des révérends Pères, 5375, avenue Notre-Damede-Grâce, Montréal 28, P.Q.

Dorge, M. Lionel, C.P. 125, Saint-Norbert, Man.

Douville, M. Raymond, Secrétariat de la Province, Québec, P.Q.

Doyon, $M^{\text {gr }}$ Paul-Emile, P.A., V.G., Evêché, C.P. 879, Trois-Rivières, P.Q.

Drolet, M. Antonio, 740, rue Monk, Québec 6, P.Q.

Drolet, M. Jean-Claude, a/s Séminaire de Chicoutimi, rue Jacques Cartier, Chicoutimi, P.Q. 
Dumais, M. le curé Charles, Sainte-Anne-de-la-Pocatière, P.Q.

Dumas, R.P. Gabriel-Marie, 4373, avenue de l'Esplanade, Montréal 18, P.Q.

Dumas, M. J.-E., 40 St. Mary's Road, Saint-Boniface, Man.

Dumouchel, M. le docteur Antoine, Rutland Hospital, Rutland, Mass. 01543

Dupont, $\mathrm{M}^{\bullet}$ J.-E., Napierville, P.Q.

Ecole Normale de Nicolet, La Sœur Directrice de l', Nicolet, P.Q.

Falardeau, M. l'abbé A., $7091-10^{\circ}$ Avenue, Ville Saint-Michel, Montréal 38, P.Q.

Filles de la Charité du Sacré-Cour de Jésus, Les, a/s Sr Rachel de Marie, 605, avenue Bowen sud, Sherbrooke, P.Q.

Forest, M. Georges-J., 183, rue Marion, Saint-Boniface, Man.

Fortin, $\mathrm{M}^{\mathrm{gr}}$ Alphonse, P.D., Séminaire de Rimouski, Rimouski, P.Q.

Foyer de Saint-Célestin, Saint-Célestin (Nicolet), P.Q.

Franciscains, Les révérends Pères, 5750, boulevard Rosemont, Montréal 36, P.Q.

Franciscains, La Bibliothèque des, 2010 ouest, boulevard Dorchester, Montréal 25, P.Q.

François-Xavier, o.s.a., Rév. Sœur, Hôtel-Dieu, Alma, P.Q.

Frenette, Son Excellence $\mathbf{M}^{\text {gr }}$ Emilien, Evêque de Saint-Jérôme, SaintJérôme, P.Q.

Frigon, Rév. Sœur Thérèse, f.c.s.p., 5655, rue de Salaberry, Montréal 9, P.Q.

Gagné, M. l'abbé A., Archevêché de Québec, C.P. 459, Québec 4, P.Q.

Gagnon, $M^{\mathrm{gr}}$ Antoine, P.A., Institut de Technologie, 25, rue Saint-Louis, Rimouski, P.Q.

Gagnon, M. l'abbé François, Collège Sainte-Anne-de-la-Pocatière, SainteAnne-de-la-Pocatière, P.Q.

Galarneau, M. Claude, 10, avenue Laurier, Québec 4, P.Q.

Gallant, Le R.P. Arthur, c.j.m., Paroisse Sainte-Amélie, C.P. 460, Baie Comeau, P.Q.

Gariépy, M. l'abbé Gérard, Berthier-en-Bas (Montmagny), P.Q.

Gaudron, R.P. E., o.f.m., Culture, 33, rue de l'Alverne, Québec, P.Q.

Gauthier, M. le docteur Dominique, Shippagan, N.-B.

Gauthier, Le R.P. Léandre, o.m.i., C.P. 361, Fort-Qu'Appelle, Sask.

Gauthier, Mme Paul-R., 605, $8^{\circ}$ Avenue, Québec 5, P.Q.

Gélineau, M ${ }^{\mathrm{gr}}$ J.-Napoléon, P.D., V.G., Archevêché d'Ottawa, 60, rue Guigues, Ottawa 2, Ont.

Gendron, M. l'abbé Paul, Côteau-Station, P.Q.

Geoffroy, M. l'abbé J.-Hector, Sainte-Emélie-de-l'Energie (Joliette). P.Q.

Germain, $\mathrm{M}^{\bullet}$ Stanislas, 72, côte de la Montagne, Québec 2, P.Q.

Gervais, Le R.P. Emile, s.j., Maison Bellarmin, 25 ouest, rue Jarry, Montréal 11, P.Q.

Giard, M. Gilles, notaire, 1555, rue Girouard, Saint-Hyacinthe, P.Q.

Giguère, R.P. Georges-Emile, s.j., 25, rue Jarry ouest, Montréal 11, P.Q.

Gingras, Mme Marcelle-G., 470, chemin Saint-Louis, Québec, P.Q. 
Gosselin, M ${ }^{\mathrm{gr}}$ Paul-Emile, P.D., 47, rue des Remparts, Québec 4, P.Q.

Goulet, M. le curé Philibert, Saint-Gervais (Bellechasse), P.Q.

Goyette, M. Armand, 19, boulevard des Erables, Iberville, P.Q.

Goyette, $\mathrm{M}^{\mathrm{gr}}$ Arsène, P.D., 10, boulevard des Erables, Iberville, P.Q.

Grand'Maison, Rév. Sœur Georgette, 280, avenue Belzile, Rimouski, P.Q.

Grand Séminaire de Québec, M. le Bibliothécaire, Québec, P.Q.

Gravel, M. l'abbé Henri, 1500, rue Orléans, Montréal 4, P.Q.

Hanzéli, M. Victor-E., 411 N.E. 81st Street, Seattle, Washington, 98105.

Harvard College Library, Record Serials, Cambridge, Mass., U.S.A. 02138

Hôpital général de Verdun, L', 4000, boulevard La Salle, Verdun, Montréal 19, P.Q.

Hudon, M. Jean-Claude, 209, rue Bossé, Chicoutimi, P.Q.

Hurtubise, R.P. Pierre, o.m.i., 249, rue Main, Ottawa, Ont.

Institution des Sourdes-Muettes, L', 3725, rue Saint-Denis, Montréal 18, P.Q.

Jésuites, Les révérends Pères, Collège de Saint-Boniface, 200, avenue de la Cathédrale, Saint-Boniface, Man.

Jésuites, Les révérends Pères, 14, rue Dauphine, Québec 4, P.Q., C.P. 188 H.V.

Joseph-Adonis, R.F., f.m.s., Scolasticat central de Montréal, 7000, rue Marie-Victorin, Montréal 39, P.Q.

Kéroack, M ${ }^{\text {rr }}$ Sylvio, P.D., Curé de la cathédrale, C.P. 336, Chicoutimi, P.Q.

Lacourcière, M. Luc, Université Laval, Québec, P.Q.

Lamirande, R.P. Emilien, o.m.i., Scolasticat Saint-Joseph, avenue des Oblats, Ottawa, Ont.

Langlois, M ${ }^{\text {gr }}$ W.-J., P.D., V.F., St Mary's Academy, Windsor, Ont.

Latourelle, $\mathrm{M}^{\mathrm{gr}}$ le curé J.-A., P.D., C.P. 740, Témiskaming, P.Q.

Lavalleee, M. l'abbé Germain, C.P. 790, Sherbrooke, P.Q.

LeBlanc, Son Excellence $\mathbf{M}^{\mathbf{g r}}$ Camille-André, Evêque de Bathurst, C.P. 460, Bathurst, N.-B.

Leblanc, $\mathbf{M}^{\bullet}$ Lionel, Protonotaire de la Cour supérieure, Palais de Justice, Saint-Hyacinthe, P.Q.

LeBlond, M. le docteur Sylvio, 341, avenue du Séminaire, Chicoutimi, P.Q.

Leclaire, M ${ }^{\text {gr }}$ J.-Charles, P.D., V.G., C.P. 218, Sorel, P.Q.

Leclerc, R.P. Jean, s.j., Université de Sudbury, Sudbury, Ont.

Lefebvre, Rév. Sœur Estelle, Hôtel-Dieu, 3840, rue Saint-Urbain, Montréal, P.Q.

Lefebvre, Jean-Jacques, archiviste en chef, Cour Supérieure, Palais de Justice, Montréal, P.Q.

Le Moignan, M. l'abbé Michel, Gaspé, P.Q.

Lemoyne, Mlle Georgette, 3950, Parc Lafontaine, Montréal, P.Q.

Lessard, M. le chanoine Léon-N., Collège de Lévis, Lévis, P.Q.

Letendre, M. J.-Antoine, Archiviste, Evêché de Nicolet, Nicolet, P.Q.

Letendre, M. le chanoine J.-G., Assistant-procureur, Archevêché de Sherbrooke, 130, rue de la Cathédrale, C.P. 4.30, Sherbrooke, P.Q.

Levesque, Son Excellence $\mathrm{M}^{\mathrm{gr}}$ Louis, Archevêque de Rimouski, Rimouski, P.Q. 
Lottinville, M. Wilfrid Lemaistre de, 548, rue Saint-Jean, Québec, P.Q. Madeleine-Durand, Rév. Sœur, f.c.s.p., Annaliste, Providence maisonmère, 5655, rue de Salaberry, Montréal 9, P.Q.

Madore, Mlle Marie-Ange, 853 est, rue Sherbrooke, Montréal 24, P.Q. Maison Provinciale Sainte-Marie, Rév. Sœurs Grises, 86, rue Ignace, Sudbury, Ont.

Malchelosse, M. Gérard, 1289, rue de la Visitation, Sainte-Foye, Québec 10, P.Q.

Malouin, Mme Reine, 1817, rue des Intendants, Québec, P.Q.

Mantha, M. Hector, 65, rue Milton, Montréal 18, P.Q.

Marie-de-la-Nativité, o.s.u., Rév. Mère, Monastère des Ursulines, C.P. 820, Roberval, P.Q.

Marie-Jeanne-de-Domrémy, Rév. Sœur, Ecole Normale, Saint-Jérôme, P.Q.

Marion, M. Séraphin, 131, rue Sunnyside, Ottawa, Ont.

Martel, M. l'abbé Clément, Presbytère Sainte-Claire, Chicoutimi-Nord, P.Q.

Martin, Son Excellence $\mathrm{M}^{\mathrm{gr}}$ Albertus, Evêché de Nicolet, Nicolet, P.Q.

Mathieu, M. le curé Aimé, Saint-Robert (Richelieu), P.Q.

Maurault, $\mathrm{M}^{\mathrm{gr}}$ Olivier, p.s.s., 346l, rue Jeanne-Mance, Montréal 18, P.Q.

Mayrand, $\mathbf{M}^{\mathrm{r}}$ Paul, P.A., Evêché, Nicolet, P.Q.

Missionnaires de l'Immaculée-Conception, Les Sœurs, Ecole secondaire Immaculée-Conception, 60, rue Saint-Joseph, Granby, P.Q.

Missionnaires de l'Immaculée-Conception, Les Sœurs, 766, rue du Cénacle, Chicoutimi, P.Q.

Missionnaires Oblats de Marie-Immaculée, Noviciat Notre-Dame, Richelieu, P.Q.

Monastère du Précieux-Sang, La Supérieure du, C.P. 186, SaintHyacinthe, P.Q.

Morisset, Fernand, 181, rue d'Youville, Sudbury, Ont.

Normand, Mme Maurice, 1120 René-Kimber, Appart. 4, Pavillon de Normandville, Trois-Rivières, P.Q.

Nourry, Le R.P. L.-M., s.m.m., 1265, rue Wyld, C.P. 718, North Bay, Ont.

O'Bready, $M^{\text {gr }}$ Maurice, P.D., Ecole Normale de L'Estrie, rue Jolliet, Sherbrooke, P.Q.

Panneton, M. le chanoine G., Couvent de Marie-Réparatrice, 2975, boulevard Laviolette, Trois-Rivières, P.Q.

Paré, Son Excellence M ${ }^{8 r}$ Marius, C.P. 278, Evêché de Chicoutimi, Chicoutimi, P.Q.

Parent, $\mathrm{M}^{110}$ Corinne-Hélène, 215, rue Somerset Ouest, Ottawa, Ont.

Parenteau, $\mathrm{M}^{\mathrm{gr}}$ Antonio, P.D., 155, rue Saint-Marcel, Drummondville, P.Q.

Petit Séminaire de Chicoutimi, La Bibliothèque du, est rue JacquesCartier, Chicoutimi, P.Q.

Picard, M. Jean-Théo., 219, rue Saint-Michel, Saint-Jean-sur-Richelieu, P.Q.

Plante, M. l'abbé Hermann, Séminaire Saint-Joseph, Trois-Rivières, P.Q.

Potvin, M. l'abbé Pascal, 53, rue Déziel, Lévis, P.Q.

Pouliot, Le R.P. Léon, s.j., Collège Sainte-Marie, 1180, rue Bleury, Montréal 2, P.Q. 
Présentation de Marie, La Supérieure provinciale des Sœurs de la, 650, rue Girouard, Saint-Hyacinthe, P.Q.

Présentation de Marie, Les Sœurs de la, C.P. 134, Acton Vale, Bagot, P.Q.

Prévost, M. Edouard, 1540, avenue Bernard, Appart. 29, Outremont, Montréal 8, P.Q.

Prince, Mme Berthe, C.P. 231, Sainte-Thérèse (Terrebonne), P.Q.

Proulx, Son Exc. $\mathrm{M}^{\mathrm{gr}}$ A., Evêque d'Alexandria, Alexandria, Ont.

Providence, La maison mère des Sœurs de la, 12055, rue Grenet, Montréal 9, P.0.

Provinciale, Rév. Mère, s.g.m., 151, rue Despins, Saint-Boniface, Man.

Provost, M. l'abbé Honorius, Séminaire de Québec, Québec, P.Q.

Raymond, M. le chanoine C.-Eugène, Curé, Saint-Pascal-de-Kamouraska, P.Q.

Raymond, M. l'abbé Marius, Presbytère Saint-Norbert, Rimouski, P.Q.

Raymond, M. Raoul, 8960, rue Saint-Denis, Montréal 11, P.Q.

Rédemptoristes, Le R.P. Recteur du monastère des, 871, rue Ontario, Sherbrooke, P.Q.

Rédemptoristes, Le Supérieur des Pères, Sainte-Anne-de-Beaupré, P.Q.

Rédemptoristes, Les révérends Pères, Sainte-Anne-des-Chênes, Man.

Richard, M. Louis, 2985, avenue de Sisson, Montréal 26, P.Q.

Robitaille, M. l'abbé Léger, 357, blvd Valcartier, Loretteville, P.Q.

Rodrigue, $\mathbf{M}^{\mathrm{gr}}$ Louis-Joseph, P.D., C.P. 100, Saint-Eustache, P.Q.

Routhier, Son Excellence $\mathrm{M}^{\mathrm{gr}}$ Henri, o.m.i., McLennan, Alta.

Roux, $\mathrm{M}^{\mathrm{gr}}$ le curé Alphonse, 245 est, rue Notre-Dame, Victoriaville, P.Q.

Roy, M. Antoine, 2050, rue Saint-Cyrille Ouest, Québec, P.Q.

Sabourin, $\mathrm{M}^{\circ}$ Roland, 370, rue Champlain, C.P. 186, Saint-Jean, P.Q.

Saint-Boniface, M. le Bibliothécaire de l'archevêché de, Saint-Boniface, Man.

Saint-Jean, M. et Mme Albert, 1028, $2^{\circ}$ avenue (Verdun), Montréal, P.Q.

Saint-Pierre, M. René, 3095, rue Girouard, Saint-Hyacinthe, P.Q.

Saint-Sacrement, Les Pères du Très, 4450, rue Saint-Hubert, Montréal 34, P.Q.

Sainte-Germaine, s.g.c., Rév. Sœur Supérieure, Hôpital Saint-Joseph, rue Mackenzie, Sudbury, Ont.

Sainte-Rose Virginie, sup. prov., Maison provinciale Bon Pasteur, 376, rue Price, Chicoutimi, P.Q.

Sainte-Theresita, s.c.i.m., Rev. Sr., Collège du Bon Pasteur, avenue Lafontaine, Chicoutimi, P.Q.

Saucier, M. le docteur Roland, C.P. 203, Chicoutimi, P.Q.

Savard, Mlle Blanche-Alice, 21, rue Price ouest, Chicoutimi, P.Q.

Savard, M. Louis, 15, rue de l'Aunier, Chicoutimi, P.Q.

Savard, M. Pierre, 860, rue Paradis, Québec 10, P.Q.

Scolasticat Saint-Charles, 455, boulevard Querbes, Joliette, P.Q.

Secrétaire générale, s.g.m., Rév. Mère, 9409, blvd Gouin ouest. Pierrefonds, P.Q.

Séminaire de Nicolet, La Bibliothèque, Nicolet, P.Q.

Séminaire du Sacré-Cœur, La bibliothèque, Station Saint-Victor (Beauce), P.Q.

Séminaire de Saint-Hyacinthe, M. le Bibliothécaire, C.P. 370, SaintHyacinthe, P.Q. 
Séminaire des Missions Etrangères, 60, rue Desnoyers, Pont-Viau, Montréal 12, P.Q.

Séminaire Saint-Jean, La Bibliothèque du, C. P. 310, Saint-Jean, P.Q.

Séminaire Saint-Thomas, M. le Bibliothécaire du, Valleyfield, P.Q.

Séminaire des Trois-Rivières, M. le Bibliothécaire du, Trois-Rivières, P.Q.

Sergerie, Mme Adéla, 14748, boul. Gouin est, Rivière-des-Prairies, Montréal 12, P.Q.

Simard, R.P. André, c.ss.r., Paroisse du Perpétuel Secours, 132, rue Blake, Hamilton, Ont.

Simard, M. l'abbé Jean-Paul, Petit Séminaire, rue Jacques-Cartier, Chicoutimi, P.Q.

Société historique de la Côte-du-Sud, La, Collège Sainte-Anne, SainteAnne-de-la-Pocatière, P.Q.

Société historique de Québec, La, Séminaire de Québec, C.P. 460, Québec 4, P.Q.

Sœurs de l'Assomption, La révérende Mère générale des, Nicolet, P.Q.

Sœurs de la Charité de Québec, La révérende Mère générale des, Ave d'Estimauville, Québec 5, P.Q.

Sœurs de la Charité, Les, Hôtel-Dieu, Saint-Hyacinthe, P.Q.

Sœurs Grises de la Croix, La Supérieure générale des, 9, rue Bruyère, Ottawa, Ont.

Sœurs de Notre-Dame du Bon-Conseil, La Maîtresse générale des Etudes des, Maison mère, Chicoutimi, P.Q.

Sœurs de Sainte-Anne, La révérende Mère générale des, Mont SainteAnne, 1950, rue Provost, Lachine, P.Q.

Sours des Saints-Noms de Jésus-Marie, La Mère provinciale des, Académie Sainte-Marie, Windsor, Ont.

Soublière, M. Hector, 240, avenue Algonquin, North Bay, Ont.

Soucy, M. le chanoine Zénon, C.P. 8, Le Bic, P.Q.

State Historical Society, The, 816 State St., Madison 6, Wisconsin, U.S.A.

Sylvain, Rév. Frère Robert, f.é.c., Académie de Québec, 2360, chemin Sainte-Foy, Québec 10, P.Q.

Tardif, R.P. Emile, o.m.i., 9916 - $110^{\circ}$ Rue, Edmonton, Alta.

Thibault, M. l'abbé Alfred, C.P. 153, Wottonville, P.Q.

Trottier, Le R.P. Aimé, c.s.c., Oratoire Saint-Joseph, Montréal 26, P.Q.

Trudeau, Le R.P. Paul-Albert, c.s.v., 1145, rue Saint-Viateur, Outremont, P.Q.

Turgeon, $\mathrm{M}^{\mathrm{gr}}$ Emile, P.D., 980, rue Richelieu, Québec 4, P.Q.

Université Laval, Division Canadiana, Bibliothèque, Québec, P.Q.

University of Chicago, Chicago, Ill., U.S.A.

Université de Sudbury, Le R.P. Recteur de l', Sudbury, Ont.

Université d'Ottawa, La Bibliothèque, Ottawa 2, Ont.

Université Pontificale Grégorienne, La Bibliothèque, Piazza della Pilotta 4, Rome, Italie.

Université Saint-Paul, La Bibliothèque, 233, rue Main, Ottawa, Ont.

Ursulines, de Rimouski, Les, Rimouski, P.Q.

Vachon, M. André, 66, rue Pie-XII, Sainte-Foy 10, Québec, P.Q.

Vachon, $\mathbf{M}^{\mathbf{g}}$ Louis-Albert, Université Laval, Québec, P.Q.

Valois, Mr Omer, P.D., V.G., Evêché, C.P. 470, Joliette, P.Q. 
Vien, l'Hon. Sénateur Thomas, suite 1506, Immeuble de la Banque Royale, 360 ouest, rue Saint-Jacques, Montréal, P.Q.

Vinet, M. Jean-Baptiste, p.s.s., 424, rue Saint-Sulpice, Montréal 1, P.Q.

Yelle, M. Gérard, p.s.s., Grand Séminaire, 2065, rue Sherbrooke ouest, Montréal 25, P.Q. 